\title{
$J$ 积 分的扩展与改进
}

\author{
尹 双 增 \\ （华北水利水电学䟚，都戦）
}

对于服从塑性形变理论的介质, J. R. Rice 证明了: 1.J 积分在物理上可解释为变形功 的差率; $2 . J$ 积分具有与积分路径无关的特性; 3.J积分可作为表示裂纹起裂的弹塑性断裂 判据: $J=J I c$.

这样, $J$ 积分避开了直接计算在裂纹尖端附近复杂的弹塑性应力应变场, 而用 $J$ 积分作为 表示裂纹尖端应变集中特征的平均参量. 因此, 十几年来, $J$ 积分成了受到普遍重视、有相当 吸引力的断裂判据, 并且得到了比较广泛的应用.

但是, 进一步的理论和实验研究表明, $J$ 积分也存在着局限性和缺陷,需要进一步研究。例 如 $J$ 积分虽然可以作裂纹起始扩展的判据, 但它只适用于有限的稳定裂纹扩展, 由于 $J$ 积分是 基于塑性形变理论而建立的,因此它不适用于有较大塑性变形裂纹扩展情况. 另外, 塑性变形 是不可逆的,因此, $J$ 积分与变形功率之间的关系不能应用于裂纹扩展过程, $J$ 积分也不能简 单地解释为裂纹扩展力, 它只可以看作是相同载荷条件下两个具有相近裂纹尺寸的同一物体 总势能的比较. $J$ 积分的这些缺陷和局限珄影响着它进一步推广和应用,必须进行深人研究.

\section{一、J积分与路径无关性的分析和扩展}

$J$ 积分的与路径无关性表明了： $J$ 积分用沿着离开裂纹尖端的路径上的应力、位移一类 状态量所决定; $J$ 积分值是裂纹尖端附近场的代表值. 于是, 如果能得到各种情况下与路径无 关的积分,则这个积分将同 $J$ 积分一样也可以直接应用到裂纹问题上.

\section{1. 与路经无关积分的求法 为了便于系统地求出与路径无关积分, 可以结合文献 $[1]$ 的} 精神提出如下的方法.

首先分析积分与路径无关性在什么条件下才能成立. 例如图 1 所示，在具有裂纹的二维 物体内, 取直角坐标系 $X_{1}, X_{2}$ 在物体内围绕 任意领域 $A$ 取一闭合路径 $\partial A$, 沿 $\partial A$ 的函数 $F$ 的积分值为

$$
\int_{\theta A} F \cdot d C=0,
$$

并且假定裂纹面上有

$$
F=0,
$$

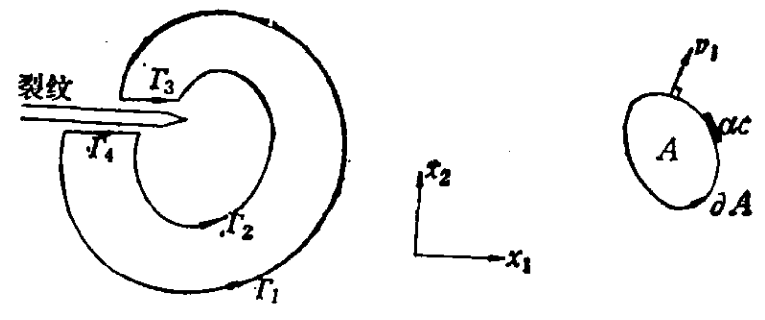

图 1 积分路径

封闭路径如图 1 所示, 即 $\Gamma_{1}+\Gamma_{3}-\Gamma_{2}+\Gamma_{4}$.

由 (1) 和 (2) 式可得到

$$
\int_{r_{1}} F \cdot d C=\int_{r_{z}} F \cdot d C,
$$

上式说明,在物体内, 函数 $F$ 沿着自裂纹下表面任一点到上表面任一点路径 $\Gamma$ 的积分值是 
不依赖于积分路径 $\Gamma$ 的,也即

$$
Q=\int_{r} F \cdot d C .
$$

可以看出, 只要能求出同时满足 (1) 式和 (2) 式的函数 $F$, 再根据此式就可以求得各种 问题的与路径无关的积分.

举例: 假设裂纹面与 $X_{1}$ 轴平行; 裂纹面上受一静水压强 $P$ 作用. 在裂纹面上 $\Gamma$ 的外法线 的单位矢量为 $\nu_{i}$, 应力矢量为 $T_{i}$, 则有

$$
\nu_{1}=0, T_{i}\left(=\sigma_{i i} \nu_{i}\right)=-P \nu_{i} .
$$

如 $G$ 为标量, $H_{i}$ 代表矢量, 则 $F$ 函数可取为

$$
F=G v_{i}+H_{i}\left(\sigma_{j i} \nu_{j}+P v_{i}\right),
$$

可见 (2) 式自动满足, 沿围绕 $A$ 域的闭合路径 $\partial A$ 取线积分时, 由 Gauss 定理有

$$
\begin{aligned}
\int_{\partial A} F \cdot d C & =\int_{\partial A}\left\{G \nu_{1}+H_{i}\left(\sigma_{j i} \nu_{i}+P v_{i}\right)\right\} d C \\
& -\int_{A}\left\{\frac{\partial G}{\partial X_{1}}+\frac{\partial H_{i}}{\partial X_{i}} \sigma_{i i}+H_{i} \frac{\partial \sigma_{j i}}{\partial X_{i}}+\frac{\partial H_{i}}{\partial X_{i}} P\right\} d A .
\end{aligned}
$$

当忽略体力和惯性力时, 因平衡方程为 $\frac{\partial \sigma_{i i}}{\partial X_{i}}=0$, 且如在物体内假定满足

$$
\frac{\partial G}{\partial X_{1}}+\frac{\partial H_{i}}{\partial X_{i}} \sigma_{i i}+\frac{\partial H_{i}}{\partial X_{i}} P=0,
$$

则由 (7) 式可以证明 (8) 式成立. 在 $J$ 积分式中, $W$ 代表应变能密度,如果

则有

$$
P=0, G-W, H_{i}=-\frac{\partial u_{i}}{\partial X_{i}},
$$

$$
G=W+P \varepsilon_{i i}, H_{i}=-\frac{\partial u_{i}}{\partial X_{i}} .
$$

看出，(8) 式也是能满足的. 如将 (9) 式及 (7) 式代入 (4) 式, 就可得到该问题的与路径 无关的积分:

$$
Q_{C}=\int_{r}\left[\left(w+P \varepsilon_{i i}\right) \nu_{1}-\left(\sigma_{i i} \nu_{i}+P \nu_{1}\right) \frac{\partial u_{i}}{\partial X_{1}}\right] d C,
$$

这样，我们就可以求得各种问题的与路径无关的积分.

2. $\boldsymbol{J}$ 积分与互余 $\boldsymbol{J}$ 积分 我们知道,具有自由表面的裂纹体的积分为

$$
J=\int_{r}\left(-w \nu_{1}-\sigma_{i i} \frac{\partial u_{i}}{\partial X_{1}}\right) d C \text {. }
$$

如裂纹表面的边界条件为

$$
\nu_{1}=0, \frac{\partial T_{i}}{\partial X_{1}}\left(=\frac{\partial \sigma_{i i}}{\partial X_{1}} \nu_{i}\right)=0,
$$

且如文献 $[2,3]$ 那样, 令 $W_{c}$ 为与 $W$ 互余的应变能密度, 并代入 (10) 式, 则可得到

$$
Q_{C}=\int_{r}\left(-w_{C} \nu_{1}+\frac{\partial \sigma_{i i}}{\partial X_{1}} u_{i} v_{1}\right) d C .
$$

比较上面的 $J$ 和 $Q_{c}$ 可以看出, $Q_{c}$ 只不过是把 $J$ 的定义式中的应为和位移交换一下位 置. 这就是说 $Q_{c}$ 和 $J$ 之间存在着某种相补关系的量.

3. 裂纹面上有外力作用时的与路经无关积分当裂纹面上有一个定值为 $\sigma_{2 i}$ 的均匀应 力矢时,也即裂纹面上的边界条件为 


$$
\nu_{1}=0, T_{i}\left(=\sigma_{i i} \nu_{i}\right)=\sigma^{B}{ }_{i} \nu_{2}
$$

时,与路径无关的积分可由下式求得

$$
\because Q=\int_{\Gamma}\left[\left(\dot{w}-\sigma_{2 i}^{B} \frac{\partial u_{i}}{\partial X_{2}}\right) \nu_{1}-\left(\sigma_{i i} \nu_{i}-\sigma_{2 i}^{B} \nu_{2}\right) \frac{\partial u_{i}}{\partial X_{1}}\right] d C,
$$

互余积分为

$$
Q_{C}=\int_{r}\left[\left(-w_{C}-a_{1 i} \sigma_{j i}\right) v_{1}+\left(u_{i}-a_{1 i} X_{2}-a_{2 i}\right) \frac{\partial \sigma_{j i}}{\partial X_{1}} \nu_{i}\right] d C,
$$

其中 $a_{i i}$ 是常数.

\section{二、 $J$ 积分作为能量释放率的分析和扩展}

作为能量释放率的 $J$ 积分的扩展，可以与路径无关的 $J$ 积分相提并论，具有同样的重要 性. 因而研究作为能量释放率的 $J$ 积分的扩展,也是弹塑性断裂力学的一个重大课题.

1. $J_{\text {oxt }}$ 积分 如果在弹性场中存在裂纹和位错缺陷，并且在其上作用有一般性的力时， 能量释放率可以用能量 - 冲量 - 张量 $P_{i} k$ 来表示:

$$
F_{k}-\int_{S} P_{i k} \nu_{i} d S \text {. }
$$

此积分是围绕缺陷的 $S$ 面来评价的; $v_{i}$ 是积分面 $S$ 的外法线单位矢. 如果用 $\boldsymbol{\delta}_{i k}$ 表 示 Chroneker $\Delta$ 系数, $W^{\circ}$ 表示弹性应变能密度,则 $P_{j k}$ 可用下式给定:

$$
P_{i k}=W^{c} \delta_{i k}-\sigma_{i j} \frac{\partial u_{i}}{\partial X_{k}},
$$

如在二维物体中有平行于 $X_{2}$ 轴的裂纹时, $F_{k}$ 则变为

$$
F_{1}=\int_{r}\left(W^{e} \nu_{1}-\sigma_{i j} \frac{\partial u_{i}}{\partial X_{1}} v_{i}\right) d C \text {. }
$$

显然, 在形式上和 Rice 的 $J$ 积分是一致的. 如果把上述结果和连续分布的位错理论结 合起来,并考虑到塑性变形的不可逆性,再把这种情况下的能量释放率公式化, 根据能量理论 观点, 就可得到参考文献 [4]提出的 Jexx:

$$
J_{\mathrm{ext}}=\int_{\Gamma}\left(W^{e} \nu_{1}-\sigma_{i j} \beta_{i i}^{c} \nu_{i}\right) d C,
$$

其中 $\beta_{i}$ 一一弹性畸变, 可由全畸变 $\frac{\partial u_{i}}{\partial X_{i}}$ 导出固有畸变 $\beta_{i i}^{*}$, 如果用连续分布位错理论时, 上式 可写为

$$
J_{\text {ext }}=\int_{0}\left(\sigma_{i j} a_{3 i}-\sigma_{i j} a_{2 i}\right) d A,
$$

其中 $a_{i j}$ 一位错密度, $Q-\Gamma$ 和裂纹所围成的领域.

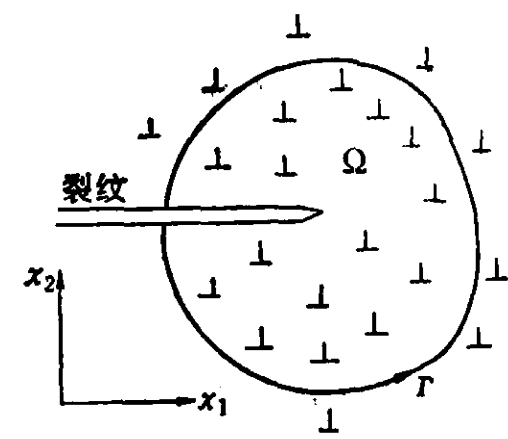

图 2 分布位错及路径

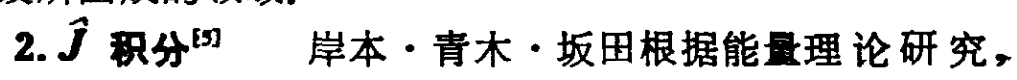
得到了流人裂纹尖端断裂进行域的能量表达式. 如图 2 所 示, 设沿包围断裂进行域的连续体的边界路径为 $\Gamma_{\text {end }}$, 则随 着裂纹扩展单位长度时, 流入断裂进行域的能量 $\hat{I}$ 为

$$
l=\int_{r_{\text {end }}} T_{i}\left(\frac{d u_{i}}{d l}\right) d C,
$$

式中 $l$ 一裂纹长度. 若用平衡式, Gauss 定理及有关假定， 则上式变为 


$$
\hat{q}-\int_{r+r_{i}}\left(W^{c} \nu_{1}-T_{i} \frac{\partial u_{i}}{\partial X_{1}}\right) d C+\int_{\lambda}\left[\sigma_{\imath} \frac{\partial \varepsilon_{i j}^{*}}{\partial X_{1}}+\left(\rho \dot{u}_{i}-F_{i}\right) \frac{\partial u_{i}}{\partial X_{1}}\right] d A,
$$

设裂纹沿 $X_{1}$ 轴扩展, $\Gamma_{s}$ 一一沿裂纹面的路径; $\dot{u}$ 一加速度; $A-\Gamma, \Gamma$, 与裂纹面所包围的区 域; $F_{i}$ 一体积力.

\section{三、一种改进的积分形式 ${ }^{[6]}$}

众所周知, Rice 提出的 $J$ 积分定义式为

$$
J=\int_{\Gamma}\left(W d X_{2}-T_{i} \frac{\partial u_{i}}{\partial X_{1}} d S\right),
$$

定义式中其它各项都有明确的物理或几何含义, 唯有应变能密度 $W$ 的物理含义不清. 因为 $J$ 积分是建立在塑性形变理论基础上的,它的主要限制条件是不允许卸载。对塑性材料、塑性变 形与应变能均与加载史有关系, 并不与应力有唯一的对应关系. 因此, 定义式中的应变能密度 $W$ 应用到弹塑性问题中还需要进一步研究.

根据上述问题，本人曾在“一种改进的 $J$ 积分形式”一文中阐述了管见，这里作一简介.

Rice 的 $J$ 积分另一种表达式为

$$
J=-\frac{\partial U}{\partial a}
$$

如取物体为单位厚度, 则 $U$ 可定义为

$$
U=\int_{A} W d A-\int_{c_{t}} T_{i} u_{i} d S,
$$

其中 $C_{t}$ 是面积为 $A$ 的物体受集中外力 $T_{i}$ 作用的部分.

对于线弹性材料,裂纹的扩展力 $G$ 为

$$
G_{I}=-\frac{\partial U}{\partial a}=\frac{k+1}{8 \mu} K_{l}^{2},
$$

式中 $\mu$ 为剪切模量; 平面应变 $k=3-4 v$, 平面应力 $k=\frac{3-v}{1+v} ; \nu$ 为泊桑比; $K_{l}$ 为应力强度 因子. 由 (24) 式知, $J$ 积分是 $G$ 的一个推广. 如果无卸载发生, 且塑性的变形理论适用, 则可 以用非线性弹性的本构关系式唯一地确定弹塑性变形. 在把 $J$ 积分应用于弹塑性裂纹问题 时,实际上已认为不可发生塑性状态的卸载现象. 因此,在这些条件下,可以用判据

$$
J=J_{I C}
$$

来预测裂纹起裂规律.

对于非线性材料, Mcclintock 指出, 裂纹尖端附近的应力应变状态是可以直接与 $J$ 积分 联系起来的, 而在非线性条件下, 利用虚功的考虑, $J$ 积分可以解释为表观的裂纹扩展力. 这 样,虚功的方程式就变为广义的 $J$ 积分. 因此, (24) 式可以看作裂纹体的一种能量平衡, $J$ 积 分代表裂纹扩展单位长度的断裂能量. 为了明确(25)式中 $W$ 的物理含意, 可以用 $\left(W^{e}+W^{p}\right)$ 代替 $W, W^{e}$ 和 $W^{p}$ 分别为弹性的和塑性的应变能密变. 这样 (24) 式就变为:

$$
J^{\prime}=\frac{\partial}{\partial a}\left[\int_{C} T_{i} u_{i} d S-\int_{A} W^{e} d A-\int_{A} W^{p} d A\right] .
$$

为了得到 $J$ 积分的常规形式, 可以利用非线性弹性材料的

$$
W^{e}=\int_{0}^{\varepsilon_{i i}^{i}} \sigma_{i j} d \varepsilon_{i j}^{e}
$$


和全应变与分应变的关系

$$
\varepsilon_{i j}=\varepsilon_{i i}^{i}+\varepsilon_{i j}^{i} .
$$

通过格林公式、平衡方程及虚功原理的推导、换算,最后得到

$$
J^{\prime}=\int_{T} W^{e} d x_{2}+\int_{A} \sigma_{i j}\left(\frac{\partial B_{i j}^{p}}{\partial x_{1}}\right) d A-\int_{\Gamma} T_{i} u_{i, 1} d S .
$$

这就是作者提出的改进的 $J$ 积分形式，我们把它书写为 $J^{\prime}$ 以示与原积分的区别. 关于 $J^{\prime}$ 积分的性质和它与积分路径选择的无关性,由下面的证明可以看出.

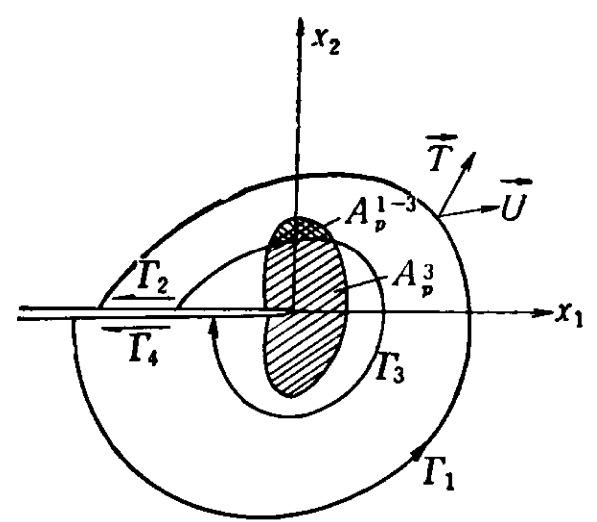

图 3

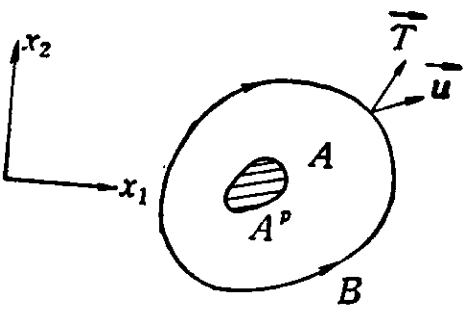

图 4

$A_{1}^{\prime}$ 为 $\Gamma_{1}$ 包含的塑性区; $A_{p}^{3}$ 为 $\Gamma_{3}$ 包含的望性区;

$A_{3}^{1-3}$ 为 $\Gamma_{1}$ 和 $\Gamma_{3}$ 之间的塑性区

如果我们取一个包含塑性区的封闭回路 $B$ ，且考虑到虚功原理：

$$
\int_{B} \vec{T} \cdot \frac{\partial \vec{u}}{\partial X_{1}} d S=\int_{A} \sigma_{i j} \frac{\partial \varepsilon_{i j}}{\partial X_{1}} d A,
$$

则沿封闭回路有

$$
L_{B}=\oint_{B}\left[W^{e} d X_{2}-T_{i} u_{i},{ }_{1} d S\right]=\int_{A}\left[\frac{\partial W^{e}}{\partial X_{1}}-\sigma_{i j} \frac{\partial \varepsilon_{i j}}{\partial X_{1}}\right] d A .
$$

利用虎克定律和复合函数的微分关系,可以得到

$$
\begin{aligned}
L_{B} & =\int_{A}\left[\sigma_{i i} \frac{\partial \varepsilon_{i j}^{e}}{\partial X_{1}}-\sigma_{i j} \frac{\partial \varepsilon_{i j}}{\partial X_{1}}\right] d A=-\int_{A} \sigma_{i j} \frac{\partial \varepsilon_{i j}^{p}}{\partial X_{1}} d A \\
& =-\int_{A_{p}} \sigma_{i j} \frac{\partial \varepsilon_{i j}^{p}}{\partial X_{1}} d A .
\end{aligned}
$$

将这一结果应用于图 3 所示封闭回路 $\Gamma=\Gamma_{1}+\Gamma_{2}+\Gamma_{3}+\Gamma_{4}$ 并设 $A_{p}^{\prime}$ 为 $\Gamma_{1}$ 包含的塑 性 区, $A_{p}^{3}$ 为 $\Gamma_{3}$ 包含的塑性区, $A_{p}^{1-3}$ 为 $\Gamma_{1}$ 和 $\Gamma_{3}$ 之间的塑性区, $A_{p}^{\prime}=A_{p}^{3}+A_{p}^{1-3}$, 则

$$
J_{\Gamma}=\oint\left[W^{e} d X_{2}-T_{i} u_{i, 1} d S\right]--\int_{A_{p}^{1-3}} \sigma_{i j} \frac{\partial \varepsilon_{i j}^{p}}{\partial X_{1}} d A \text {. }
$$

注意在封闭回路上 $\Gamma_{2}$ 和 $\Gamma_{4}$ 为裂纹自由表面，其上无外力作用，即 $T_{i}-0$, 使 $J$ 积分式左 端第二项为零,并且由于是沿 $X_{1}$ 轴方向, $d X_{2}=0$, 使第一项也为零. 因此, $\Gamma_{2}$ 和 $\Gamma_{4}$ 的积分 均为零, 而 $\Gamma_{1}$ 和 $\Gamma_{3}$ 的方向相反, 由此得到

$$
J_{r}=\int_{r_{1}}\left[W^{e} d X_{2}-T_{i} u_{j, 1} d S\right]-\int_{r_{3}}\left[W^{e} d X_{2}-T_{i} u_{i, 1} d S\right]
$$




$$
=-\int_{A_{p}^{1-3}} \sigma_{i i} \frac{\partial \varepsilon_{i j}^{p}}{\partial X_{1}} d A,
$$

因为 $A_{p}^{1-3}-A_{p}^{1}-A_{p}^{3}$, 所以

由此得到

$$
\begin{gathered}
\int_{r_{1}}\left[W^{e} d X_{2}-T_{i} u_{i, 1} d S\right]-\int_{r_{3}}\left[W^{e} d X_{2}-T_{i} u_{i, 2} d S\right] \\
\left.--\iint_{A_{p}^{1}} \sigma_{i j} \frac{\partial \varepsilon_{i 1}^{p}}{\partial X_{1}} d A-\int_{A_{p}^{3}} \sigma_{i j} \frac{\partial \varepsilon_{i j}^{p}}{\partial X_{1}} d A\right] \\
=-\int_{A_{p}^{1}} \sigma_{i j} \frac{\partial \varepsilon_{i j}^{p}}{\partial X_{1}} d A+\int_{A_{p}^{3}} \sigma_{i j} \frac{\partial \varepsilon_{i i}^{p}}{\partial X_{1}} d A,
\end{gathered}
$$

$$
\begin{aligned}
& \int_{r_{1}}\left[W^{e} d X_{2}-T_{i} u_{i, 1} d S\right]+\int_{A_{p}^{\frac{1}{2}}} \sigma_{i j} \frac{\partial \varepsilon_{i j}^{p}}{\partial X_{1}} d A \\
& =\int_{r_{3}}\left[W^{e} d X_{2}-T_{i} u_{i, 1} d S\right]+\int_{A_{p}^{3}} \sigma_{i j} \frac{\partial \varepsilon_{i j}^{p}}{\partial X_{1}} d A .
\end{aligned}
$$

这就证明了 (31) 式 $J^{\prime}$ 积分值和积分路径选择无关.

为了说明 $J^{\prime}$ 积分的适用范围，我们可以将 $J^{\prime}$ 积分和原 $J$ 积分加以比较. 在 $J^{\prime}$ 积分式中, 既包含了弹性部分也包含了塑性部份. 众所周知, 对于理 想的弹性材料, 塑性应变 $\varepsilon_{i i}^{p}=0$; 材料加载时储存的弹性 应变能 $W$ 等于材料卸载时释放出来的弹性应变能 $W^{e}$, 即

$$
W=W^{e} \text {, }
$$

这样 (31) 式就变为

$$
\begin{aligned}
J^{\prime} & =\int_{\Gamma} W^{e} d X_{2}+\int_{A} \sigma_{i j}\left(\frac{\partial \varepsilon_{i j}^{p}}{\partial X_{1}}\right) d A-\int_{r} T_{i} u_{i, 1} d S \\
& =\int_{\Gamma} W d X_{2}-\int_{\Gamma} T_{i} u_{i}, 1, d S,
\end{aligned}
$$

即 $J^{\prime}=J$. 这说明, 原 $J$ 积分只适用于理想弹性材料，而 $J^{\prime}$ 积分可以适用于弹塑性材料; $J$ 积只能预测裂纹的起始 开裂，而 $J^{\prime}$ 积分还能预测裂纹的亚临界扩展以至失稳扩展 的整个过程. 由图 5 所示的计算与实验结果比较也可看出 二者的趋势和数值均吻合较好. 图 5 中表示的是 $J^{\prime}$ 积分

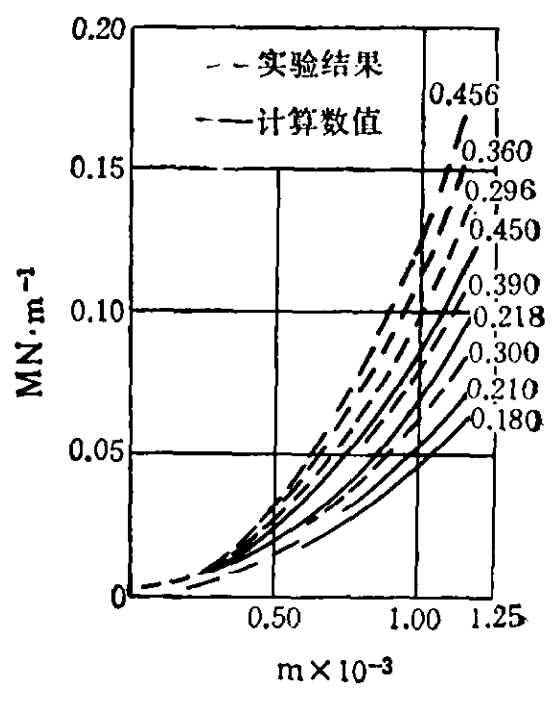

图 5 对试件的位移曲线. 用 400 号钢板中心裂纹进行实验. 图中的数字表示总的裂纹长度.

\section{考文嗝}

[1] 大路清閶・久保司郎,日本机珹学会论文集, 69(1978), 101.

[2] Begley, J. A. and landes, J. D., ASTMSTP, 514 (1972), 1-32.

[3] Sailors, R. H. and Corten, H. T., ASTMSTP, 514(1972), 164-191.

[4] Hutchinson, J.W., J., Mech. Phys, Solids, 16(1968), 13.

[5] 岸本軎久雄等, 日本机珹学会论文集, 46(1980), 1049.

[6] 尹双增, 北方七省市力学学术年会论文集, 1986,41. 